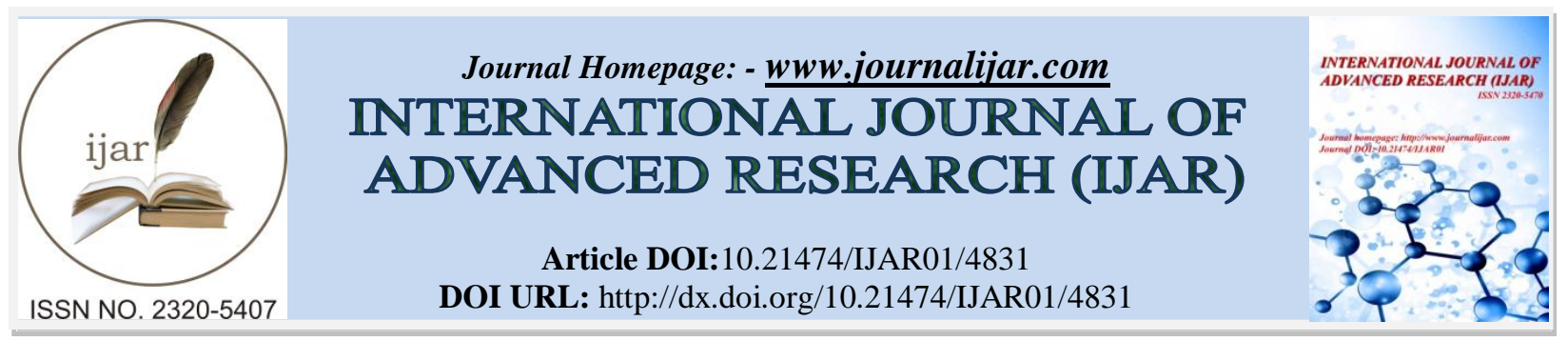

RESEARCH ARTICLE

\title{
EFFECT OF ALCOHOLIC AND NON ALCOHOLIC MOUTHWASHES ON PRE-STRETCHED ELASTOMERIC CHAIN.
}

\begin{abstract}
Dr. Shameem Hassainar, Dr. Thimmiah U. K, Dr. Chetan G. B, Dr. Shashwath M. S , Dr. Veena S, Dr. Kiran
\end{abstract} Kumar K , Dr. Smitha P.

\section{Manuscript Info}

Manuscript History

Received: 12 May 2017

Final Accepted: 14 June 2017

Published: July 2017

Key words:-

Elastomeric chain, force decay, mouthwash

\section{Abstract}

Background: Elastomeric chains are commonly used in orthodontics. Force decay in these materials poses clinical problems. The aim of this study was to evaluate the effects of three different mouthwashes on the force decay of orthodontic chains.

Materials and Methods: In this experimental study, Elastomeric chain of clear continuous type was used.The media used for the study was grouped into 3 groups.

The first group (Group A)served as Control group (Deionised water). Second group (Group B) consisted of three alcohol containing mouthwashes of varying alcohol content from $21.6 \%$ to $6 \%$ alcohol. The Third group (Group C) consisted of three non alcoholic mouthwashes .The Control group (Group A) consisted of Deionized (DI) water.

The specimens were then stretched to $23 \mathrm{~mm}$ and mounted between the rows of the stainless steel pins on the acrylic jig. The elastomeric chains belonging to each sample groups were pre-stretched $(\times 2$ times) $(100 \%)$ and $(150 \%)$ in Sample-A and Sample-B respectively in Jig-B of the initial length of elastomeric chain prior to mounting on the pins in Jig-A.

Results: Comparisons were made between the Groups both IntraGroup and Inter-Group. The Inter-Group comparison of force decay was done within the groups namely Sample A and Sample B respectively. The Inter-Group comparison of force decay was done within the groups of each sample test groups.

Conclusion: The force decay patterns of Control Deionsed water group and Non Alcoholic Mouth rinses group in 150\% pre-stretched e-chain was comparable and prescription of Non Alcoholic mouthwashes seems optimum for hygiene maintenance as compared to Alcoholic mouth rinses in order to have the least effect on force decay of elastomeric chains.

Copy Right, IJAR, 2017,. All rights reserved.

\section{Introduction:-}

Elastomeric chains generate light continuous forces for canine retraction, diastema closure, rotation correction and arch constriction ${ }^{1}$. 
An elastomer ${ }^{2,3}$ is any rubbery material composed of long chainlike molecules or polymers that are capable of recovering their original shape after being stretched to great extents-hence the name elastomer, from "elastic polymer".

The Elastomer with the longest history of use is poly-isoprene, the polymer constituent of natural rubber. Elastomers have an internal flexibility and highly elastic. On release from being extended, they must be able to return spontaneously to a disordered state by random motion of their repeating units ${ }^{2}$.Synthetic rubber polymers developed from petrochemicals in the 1920's have a weak molecular attraction consisting of primary and secondary bonds. On extension these molecular chains unfold in an orderly linear fashion. Elastomeric chains are however not without their disadvantages. When exposed to oral environment, they absorb water and saliva, permanently stain and suffer a breakdown of internal bonds that leads to permanent deformation. This breakdown and deformation produces force decay in the elastomeric chains as the day's progress from its initial application. This loss of force due to stress relaxation results in a gradual loss of effectiveness. Various factors have been shown to impact the amount of force decay observed with elastomeric chains. In 1975, Hershey and reynolds5 noted a 50\% loss of force after the first day with $40 \%$ of original force remaining after 4 weeks. In dry air, the percentage of force decay after 21 days was illustrated to be $42 \%$ to $63 \%$.In addition, force decay occurred more quickly when Elastomers were submerged in a water bath. The greatest degree of force loss occurred in the first 3 hours with remainder of force decay staying relatively constant over the next 21 days.

In order to better simulate the oral environment, thermal cycling of water bath in which elastomers were submerged were tested 9 . A decreased percentage of force decay was demonstrated at 30 minutes (23\% to $37 \%$ ) and after 21 days $(39 \%$ to61\%). Additionally, tooth movement was simulated by decreasing the stretch distance of the elastomeric chain by $0.5 \mathrm{~mm} /$ week, while following the same thermal cycling protocol ${ }^{10}$.Compared to the thermally cycled control; this resulted in a significantly higher amount of force decay at each time interval ending with (51\% to $68 \%$ ) decay at 21 days.

The consensus of various studies is that force decay of elastomeric materials occurs more rapidly in vivo than in vitro, no conclusions have been established as to what causes this difference even when attempts to simulate oral environment were made. The factors such as temperature and $\mathrm{pH}$ have been studied but remains

Controversial However recently due to concerns with regards to alcoholic content and its possible adverse effects , non alcoholic mouth rinses have started to enter the market and it is assumed that the higher force decay of elastomeric chains can be avoided if patients are prescribed non alcoholic mouth rinses.

Therefore the need of this present study is to evaluate and compare the force decay properties of pre-stretched elastomeric chains in alcoholic and non alcoholic mouth rinses in order to ascertain more favorable mouth rinse for orthodontic patients thatwould have the least effect on the force decay of elastomeric chains

\section{Aims and Objectives:-}

$>$ To assess whether there is force decay in controlled environment.

$>$ To assess the effects of alcoholic mouthwashes and how different concentration of alcohol in different mouthwashes affects the force decay in a pre-stretched elastomeric chains.

$>$ To compare the effects of non alcoholic and alcoholic mouthwashes with regards to their effects on force degradation in a pre-stretched elastomeric chains.

$>$ To ascertain a favorable mouthwash for orthodontic patients that would have the least impact on the force applied by the Elastomeric chains.

\section{Materials and Methods:-}

\section{Materials:-}

Elastomeric chain of clear continuous type manufactured by Ortho Organizers was selected for testing .The media used for the study was grouped into 3 groups.

The first group (Group A)served as Control group (Deionised water). Second group (Group B) consisted of three alcohol containing mouthwashes of varying alcohol content from $21.6 \%$ to $6 \%$ alcohol. The Third group (Group C) consisted of three non alcoholic mouth washes. The Control group (Group A) consisted of Deionized (DI) water. 


\title{
Equipments used in the study:-
}

Jigs:-

JIG-A- Clear Acrylic blocks measuring $18 \mathrm{~cm} \times 6 \mathrm{~cm} \times 7 \mathrm{~mm}$ was cut. The blocks had two rows of fifteen stainless steel pins each of $0.040 \mathrm{~mm}$ diameter which were stabilized $23 \mathrm{~mm}$ apart.

JIG-B- Clear acrylic block measuring $18 \mathrm{~mm} \times 6 \mathrm{~mm} \times 7 \mathrm{~mm}$ was cut . The block had two rows of four stainless steel pins each of diameter $0.040 \mathrm{~mm}$ diameter which were stabilized at $24 \mathrm{~mm}(100 \%)$ and $30 \mathrm{~mm}(150 \%)$ respectively.

The elastomeric chains were pre-stretched to $100 \%$ and $150 \%$ length than the initial length in JIG-B. Pre-stretched elastomeric chains are further stretched between the stainless steel i.e. $23 \mathrm{~mm}$ apart in JIG-A in each case and force was measured each time. The elastomeric chains were immersed in various mouthwashes(Group B and Group C) and Deionised water(Control Group A) .The elastomeric chains was immersed 60 seconds twice a day in morning and evening in the mouthwash media for both Group B and Group C.

\section{Materials used are:-}

Universal Testing Machine (International Equipments, Bombay, India).(Fig .14),Digital Vernier Caliper (Aero Space, Bangalore, India).Incubator (Heat control Instruments and Services, Bangalore, India),Clock/Timer ,Thermometer.

\section{Methodology:-}

The orthodontic elastomeric chains were immersed in Control Group A (Deionizedwater) (Media A). (Fig.17)

And 3 mouth rinses in the Alcoholic group (Group B) which was namely:

$>$ Oragard Mouth rinse containing 21.6\% v/v Alcohol (Media B1)

$>$ Lidocam Mouth rinse containing 10\% v/v Alcohol (Media B2)

$>$ Pepsodent Germicheck Mouth rinse containing 6\% v/v alcohol (MediaB3)

\author{
And 3 mouth rinses in the Non-Alcoholic Group (Group C) which was namely:- \\ $>$ Colgate Plax Freshmint mouth rinse(Media C1). \\ $>$ Hiora-k Mouth rinse (Media C2) \\ $>$ Colgate Plax Sensitive mouth rinse (Media C3).
}

Each Jig had 15 rows of stainless steel pins stabilized $23 \mathrm{~mm}$ apart. Elastomeric chains were stretched between the stainless steel pins (23 mm apart). Each 5 link clear elastomeric chain measuring $12 \mathrm{~mm}$ at initial length was considered as a specimen. Each study sample groups i.e.; Sample A- 100\% stretch group and Sample B- 150\% stretch group have specimens was stretched between the stainless steel pins and held between the pins at a constant length. 15 specimens were stretched and held between each pair of pins which made a total of 105 specimens (Group A, B and C) with a total of 525 specimens for the 7 test groups in each sample groups. A total of 1050 specimens are present in both sample groups combined. Each of 5 link clear continuous elastomeric chains were measured using a Digital Vernier caliper and their initial length was noted as $12 \mathrm{~mm}$ which is approximately $50 \%$ of the $23 \mathrm{~mm}$ distance between the canine bracket hook and molar tube hook in an average arch and their force was measured and recorded. The specimens were then stretched to $23 \mathrm{~mm}$ and mounted between the rows of the stainless steel pins on the acrylic jig. The elastomeric chains belonging to each sample groups were pre-stretched $(\times 2$ times $)$ $11,19,36(100 \%)$ and (150\%) in Sample-A and Sample-B respectively in Jig-B of the initial length of elastomeric chain prior to mounting on the pins in Jig-A to bring down the force levels to within physiologic limits which is estimated to range from as low as $100 \mathrm{gms}$ to as high as $350 \mathrm{gms} 10$ The Fourteen test sample Jigs were independently submersed in Deionized water and incubated at $37^{\circ}$ Celsius in an Incubator and monitored everyday with a thermometer16. Six test sample jigs from both sample groups (100\%-3nos and 150\%-3nos.) were immersed in Media Group B( Media B1, B2 and B3) containing alcoholic mouthwash mixed with Deionized water in the ratio of 2:1 for 60 seconds as $20 \mathrm{ml}$ quantity of mouthwash is recommended to be swirled in the mouth for 1 minute (60 Seconds) twice a day18 and six test sample jigs(100\%-3nos and 150\%-3nos.) were immersed in Media Group C( Media C1, C2 and C3) containing Non Alcoholic mouthwash mixed with Deionized water in the ratio of 2:1 for 60 seconds twice a day and force was measured with Ingstron Universal Testing Machine Each 60 second exposure was measured using a timer and two daily exposures were separated by 9 hours. The control group (Group A) underwent the same protocol; however, these specimens were only exposed to DI water (Media A). 
The force was measured at the following time intervals: Initial (0), 1 Hour, 7th day, 14th day, 21st day and 28 th day. Force measurements were made with Universal testing Machine. The 5 link elastomeric chain was mounted on the Universal testing machine with the help of rigid steel wires which was looped from the terminal ends of the 5 link chain and secured to the testing machine .Measurement readings were taken with elastomeric chain stretched to the same $23 \mathrm{~mm}$ length that the jig pins had previously maintained them. Between measurements the seven jigs were immersed in Deionized water and stored in incubator at $37^{\circ}$ Celsius for the test period of 28 days. After the 28 th day, the data was analyzed statistically using Paired t-test, Descriptive analysis, Repeated Measure ANOVA to study and compare the influence of the aforesaid mouth rinses on the force decay of pre-stretched elastomeric chains .All the statistical methods were carried out through the SPSS for Windows (version16.0)

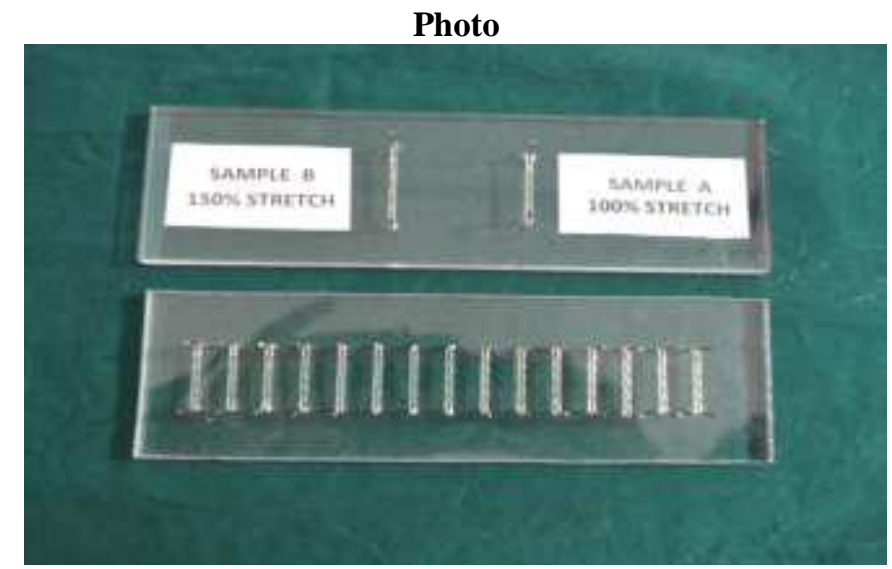

Jig with E-chain pre-stretched to $100 \%$ and $150 \%$

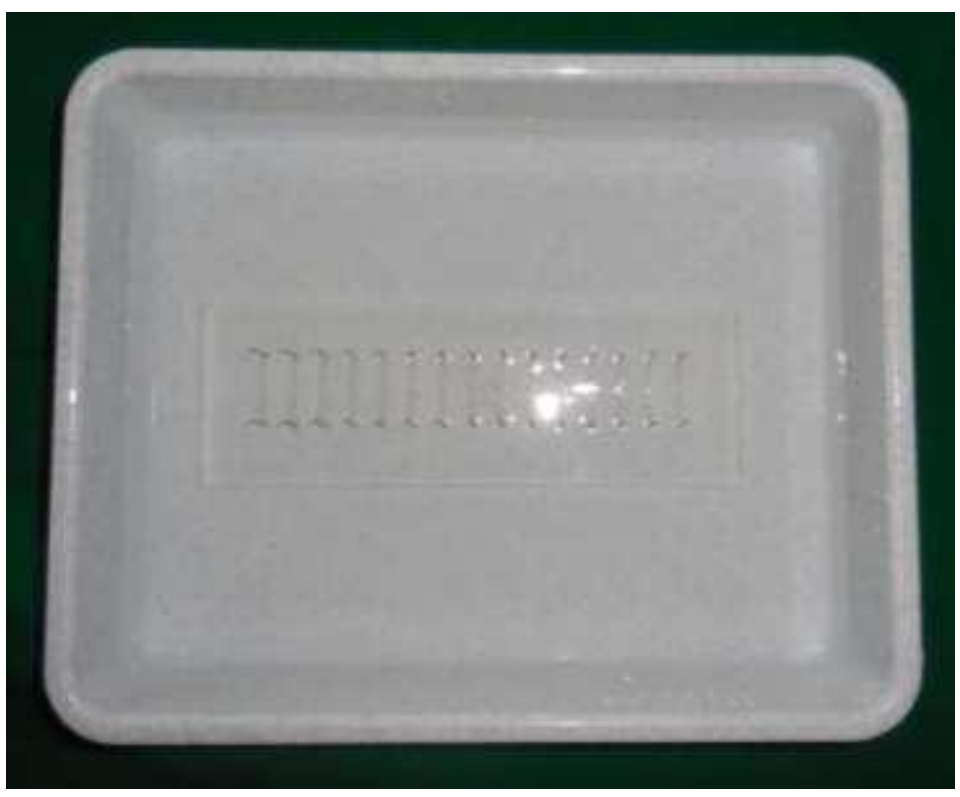

Control Group A (Deionized water) (Media A). 


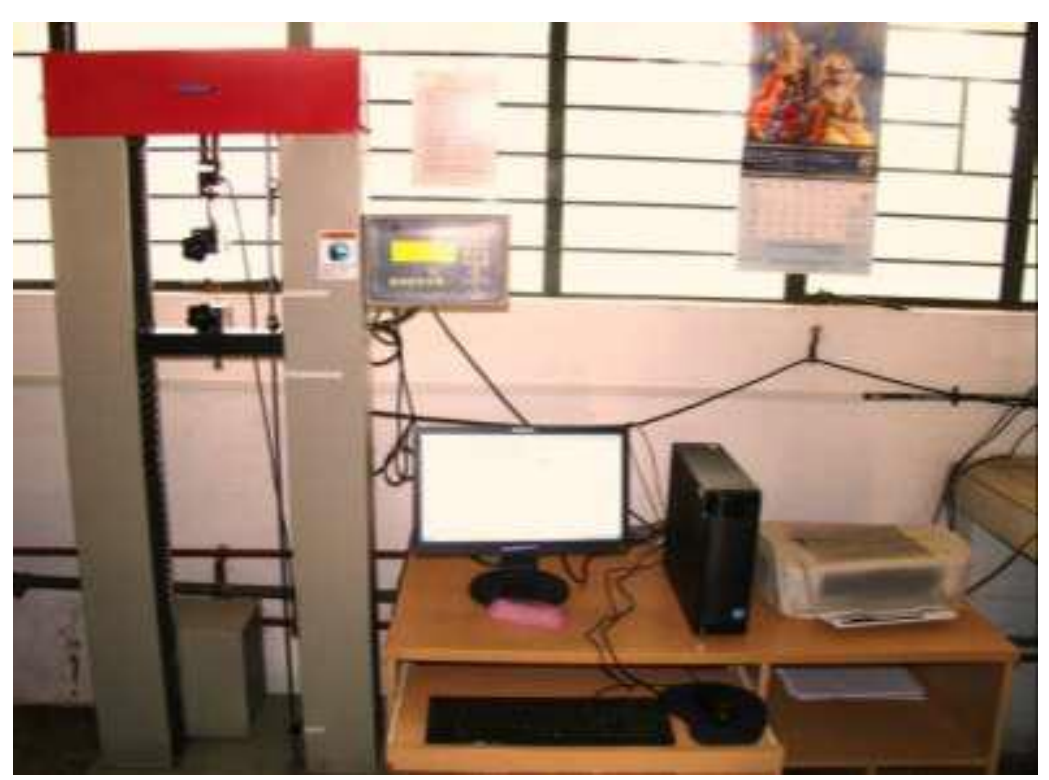

Ingstron Universal Testing Machine

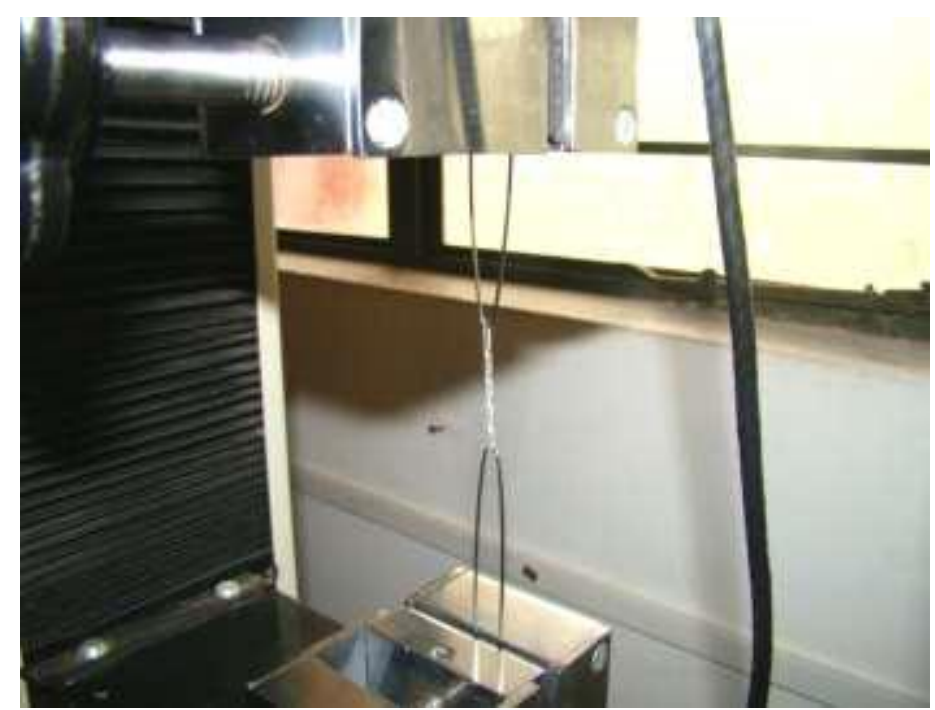

E-chain being stretched in Universal Testing Machine

\section{Results:-}

The Control Group comprised of Deionised (DI) water whereas the test group comprised of three commercially available Alcoholic mouth rinses of Varying concentration of Alcohol ( Oragard, Lidocam and Pepsodent Germi check) and three commercially available Non Alcoholic mouth rinses (Colgate Plax Fresh mint, Hiora-k and Colgate Plax sensitive) mixed with DI water in a ratio of $2: 1$ incubated at $37^{\circ} \mathrm{C}$.

The force decay was measured at six time points (Initial Zero Hour, 1 hour, 1week, 2 week, 3 week, and 4 week)

There were no significant differences between the groups at the Initial time point (Zero hour) $(p=0.098)$

Comparisons were made between the Groups both Intra-Group and Inter-Group.

The Inter-Group comparison of force decay was done within the groups namely Sample A and Sample B respectively.

The Inter-Group comparison of force decay was done within the groups of each sample test groups. 


\section{Inter Group comparison between Alcoholic groups:-}

No differences were noted in the force decay properties between the Alcohol oragard, Alcoholic lidocam and Alcoholic pepsodent group at any time point in both sample A and Sample B. However, Alcohol pepsodent group showed statistically significant difference at the end of 4 weeks compared to Alcohol lidocam and Alcohol oragard group in both Sample A and Sample B test groups (Graph 1 and 2)

\section{Inter Group comparison between the Non Alcoholic groups:-}

No differences in force decay were demonstrated between the Non Alcoholic Fresh mint, Non Alcoholic Hiora and Non Alcoholic plax groups at any time point in both samples (A\&B) (Graph 1 and 2).However, Hiora-k showed statistically significant difference at the end of 4th week compared to Non alcoholic Freshmint and Non-alcoholic plax groups in both Sample A and Sample B test groups.

Inter Group comparison between Alcoholic Group and Non Alcoholic group:-

Statistically significant differences were obtained in the force decay between the Alcoholic group and the Non Alcoholic Group at all time points. (Graph1 and 2).Here Non Alcoholic groups had lesser force decay compared to Alcoholic groups in both Sample A and Sample B.

However in Sample A test group; Non-alcoholic Hiora group had least force decay than the rest of the groups and Alcoholic Oragard group with the highest force decay. In sample B test groups; Non alcoholic Freshmint group had least force decay than the rest of the groups and Alcoholic Oragard group with the highest force decay.

Intergroup comparison between Control and Alcoholic group:-

Statistically significant differences was demonstrated between the Control DI water group and Alcoholic group with the Alcoholic group demonstrating Increased force decay at each time points and Significantly less residual force remaining at the end of 4th week as compared to DI water control group in both Sample A and Sample B.(Graph 1 and 2)

Intergroup comparison between Control and Non Alcoholic group:-

No differences in force decay was demonstrated between the control Group and the Non Alcoholic group at all time points $(\mathrm{P}=0.548)$.In Sample A test groups from the3rd week to the 4th week it was noted that force decay in Non Alcoholic group was slightly more than the DI water Control group with Non Alcoholic group demonstrating slightly lower residual force at the end of 4th week than DI control group. (Graph 1 and 2). Where as in Sample B test groups, the Non Alcoholic groups showed difference in force decay from 2nd week to the end of 4th week which was significantly less force decay when compared to control DI water control groups.

\section{Inter group comparison between Sample A and Sample B test groups:-}

No differences in force decay were demonstrated between the Sample groups at any time point ( Graph 3).However, Sample A showed statistically significant difference from 3rd to the end of 4th week compared to its counterpart Sample B, i.e. ; Sample A showed higher force decay than Sample B test groups.

TABLE 1: Force Measurements in Gms of Each group over 4 weeks in Sample A

\begin{tabular}{|c|c|c|c|c|c|c|c|}
\hline VARIANT & DI WATER & $\begin{array}{c}\text { ALCOHOL } \\
\text { ORAGARD }\end{array}$ & $\begin{array}{c}\text { ALCOHOL } \\
\text { LIDOCAM }\end{array}$ & $\begin{array}{c}\text { ALCOHOL } \\
\text { PEPSODENT }\end{array}$ & $\begin{array}{c}\text { NON } \\
\text { ALCOHOLIC } \\
\text { FRESHMINT }\end{array}$ & $\begin{array}{c}\text { NON } \\
\text { ALCOHOLIC } \\
\text { HIORA }\end{array}$ & $\begin{array}{c}\text { NON } \\
\text { ALOHOLIC } \\
\text { PLAXX }\end{array}$ \\
\hline INTIAL & $360.36=0.38$ & $358.44 \pm 0.30$ & $358.4=0.33$ & $359.48 \pm 0.35$ & $358.54 \pm 0.35$ & $359.50 \pm 0.36$ & $358.52 \pm 0.29$ \\
\hline 1 HOUR & $282.47=0.45$ & $255.55 \pm 0.26$ & $259.36=0.35$ & $264.52 \pm 0.29$ & $277.46 \pm 0.38$ & $281.57 \pm 0.32$ & $278.56 \pm 0.34$ \\
\hline 1 WEEK & $264.50=0.30$ & $222.45 \pm 0.41$ & $225.51=0.41$ & $228.4 \pm 0.29$ & $257.65 \pm 0.37$ & $259.53 \pm 0.31$ & $259.50 \pm 0.45$ \\
\hline 2 WEEK & $232.46=0.40$ & $190.46 \pm 0.31$ & $196.44=0.47$ & $197.48 \pm 0.33$ & $236.38 \pm 0.46$ & $229.47 \pm 0.34$ & $235.63 \pm 0.30$ \\
\hline 3 WEEK & $203.55=0.40$ & $152.61 \pm 0.34$ & $156.47=0.43$ & $158.44 \pm 0.26$ & $195.51 \pm 0.34$ & $199.56 \pm 0.35$ & $197.51 \pm 0.37$ \\
\hline 4 WEER & $170.58=0.40$ & $109.54 \pm 0.42$ & $115.47=0.41$ & $114.61 \pm 0.19$ & $154.41 \pm 0.39$ & $165.60 \pm 0.39$ & $157.59 \pm 0.38$ \\
\hline
\end{tabular}


Table 2: Percentage of force decay of Each Group over 4 weeks in Sample A

\begin{tabular}{|c|c|c|c|c|c|c|c|}
\hline VARIANT & $\begin{array}{c}\text { DI } \\
\text { WATER }\end{array}$ & $\begin{array}{c}\text { ALCOHOL } \\
\text { ORAGARD }\end{array}$ & $\begin{array}{c}\text { ALCOHOL } \\
\text { LIDOCAM }\end{array}$ & $\begin{array}{c}\text { ALCOHOL } \\
\text { PEPSODENT }\end{array}$ & $\begin{array}{c}\text { NON } \\
\text { ALCOHOLIC } \\
\text { FRESHMINT }\end{array}$ & $\begin{array}{c}\text { NON } \\
\text { ALCOHOLIC } \\
\text { HIORA }\end{array}$ & $\begin{array}{c}\text { NON } \\
\text { ALCOHOLIC } \\
\text { PL.AX }\end{array}$ \\
\hline INTIAL & $100 \%$ & $100 \%$ & $100 \%$ & $100 \%$ & $100 \%$ & $100 \%$ & $100 \%$ \\
\hline 1 HOUR & $78.3 \%$ & $71.2 \%$ & $72.5 \%$ & $73.7 \%$ & $77.2 \%$ & $78.2 \%$ & $77.4 \%$ \\
\hline 1 WEER & $73.3 \%$ & $61.9 \%$ & $62.8 \%$ & $63.5 \%$ & $71.7 \%$ & $72.1 \%$ & $72.1 \%$ \\
\hline 2 WEER & $64.5 \%$ & $53.1 \%$ & $54.8 \%$ & $54.9 \%$ & $65.8 \%$ & $63.7 \%$ & $65.7 \%$ \\
\hline 3 WEEK & $56.3 \%$ & $42.6 \%$ & $43.6 \%$ & $44.3 \%$ & $54.4 \%$ & $55.4 \%$ & $55.0 \%$ \\
\hline 4 WEEK & $47.3 \%$ & $30.6 \%$ & $32.19 \%$ & $34.18 \%$ & $43.02 \%$ & $46.08 \%$ & $43.9 \%$ \\
\hline
\end{tabular}

TABLE 3:Force Measurements in Gms of Each group over 4 weeks in Sample B

\begin{tabular}{|c|c|c|c|c|c|c|c|}
\hline VARIANT & DI WATER & $\begin{array}{l}\text { ALCOHOL } \\
\text { ORAGARD }\end{array}$ & $\begin{array}{l}\text { ALCOHOL } \\
\text { LDOCAM }\end{array}$ & $\begin{array}{l}\text { ALCOHOL } \\
\text { PEPSODENT }\end{array}$ & $\begin{array}{l}\text { NON } \\
\text { ALCOHOLIC } \\
\text { FRESHMINT }\end{array}$ & $\begin{array}{c}\text { NON } \\
\text { ALCOHOLIC } \\
\text { HORA }\end{array}$ & $\begin{array}{l}\text { NON } \\
\text { ALCOHOLIC } \\
\text { PL.AX }\end{array}$ \\
\hline INITIAL & $360.37 \pm 04$ & $358.48=0.30$ & $358.47=0.25$ & $359.45=0.43$ & $358.55=0.27$ & $359.46 \pm 0.42$ & $358.49 \pm 0.39$ \\
\hline 1 HOUR & $240.51=0.42$ & $206.52=0.34$ & $242.47=0.37$ & $286.41=0.38$ & $288.51=0.30$ & $292.50=0.28$ & $298.58 \pm 0.40$ \\
\hline 1 WEEK & $220.50=0.31$ & $192.57=0.42$ & $220.61=0.32$ & $244.48=0.31$ & $262.69=0.32$ & $264.57 \pm 0.36$ & $269.49 \pm 0.47$ \\
\hline 2 WEEK & $201.52=0.27$ & $170.45=0.26$ & $191.30=0.25$ & $211.45=0.25$ & $245.43=0.31$ & $243.52 \pm 0.34$ & $254.65 \pm 0.28$ \\
\hline 3 WEEK & $175.60=0.33$ & $132.63=0.25$ & $143.57=0.34$ & $178.46=0.35$ & $223.47=0.31$ & $216.55 \pm 0.32$ & $227.53 \pm 0.28$ \\
\hline 4 WEEK & $143.59=0.31$ & $101.50=0.36$ & $109.57=0.26$ & $138.58=0.34$ & $196.59=0.35$ & $184.49 \pm 0.28$ & $186.56 \pm 0.36$ \\
\hline
\end{tabular}

Table 4: Percentage of force decay of Each Group over 4 weeks in Sample B

\begin{tabular}{|l|c|c|c|c|c|c|c|}
\hline VARIANT & $\begin{array}{c}\text { DI } \\
\text { WATER }\end{array}$ & $\begin{array}{c}\text { ALCOHOL } \\
\text { ORAGARD }\end{array}$ & $\begin{array}{c}\text { ALCOHOL } \\
\text { LIDOCAM }\end{array}$ & $\begin{array}{c}\text { ALCOHOL } \\
\text { PEPSODENT }\end{array}$ & $\begin{array}{c}\text { NON } \\
\text { ALCOHOLIC } \\
\text { FRESHMINT }\end{array}$ & $\begin{array}{c}\text { NON } \\
\text { ALCOHOLIC } \\
\text { HIORA }\end{array}$ & $\begin{array}{c}\text { NON } \\
\text { ALOHOLIC } \\
\text { PLAX }\end{array}$ \\
\hline INITLAL & $100 \%$ & $100 \%$ & $100 \%$ & $100 \%$ & $100 \%$ & $100 \%$ & $100 \%$ \\
\hline 1 HOUR & $66.8 \%$ & $57.7 \%$ & $67.7 \%$ & $79.7 \%$ & $80.3 \%$ & $81.2 \%$ & $83.1 \%$ \\
\hline 1 WEER & $61.09 \%$ & $53.6 \%$ & $61.5 \%$ & $68.0 \%$ & $73.2 \%$ & $71.9 \%$ & $75.04 \%$ \\
\hline 2 WEER & $55.8 \%$ & $47.5 \%$ & $54.09 \%$ & $58.9 \%$ & $68.4 \%$ & $67.7 \%$ & $70.9 \%$ \\
\hline 3 WEER & $48.6 \%$ & $36.9 \%$ & $40.0 \%$ & $49.7 \%$ & $62.2 \%$ & $60.2 \%$ & $63.3 \%$ \\
\hline 4 WEER & $39.8 \%$ & $28.2 \%$ & $30.5 \%$ & $38.5 \%$ & $54.8 \%$ & $51.2 \%$ & $52.3 \%$ \\
\hline
\end{tabular}


Graph 1:- Force Decay Patterns (In Gms) Observed When Comparisons Were Done Between The Test Groups (Intra Group Comparison) Of both Sample A and Sample B

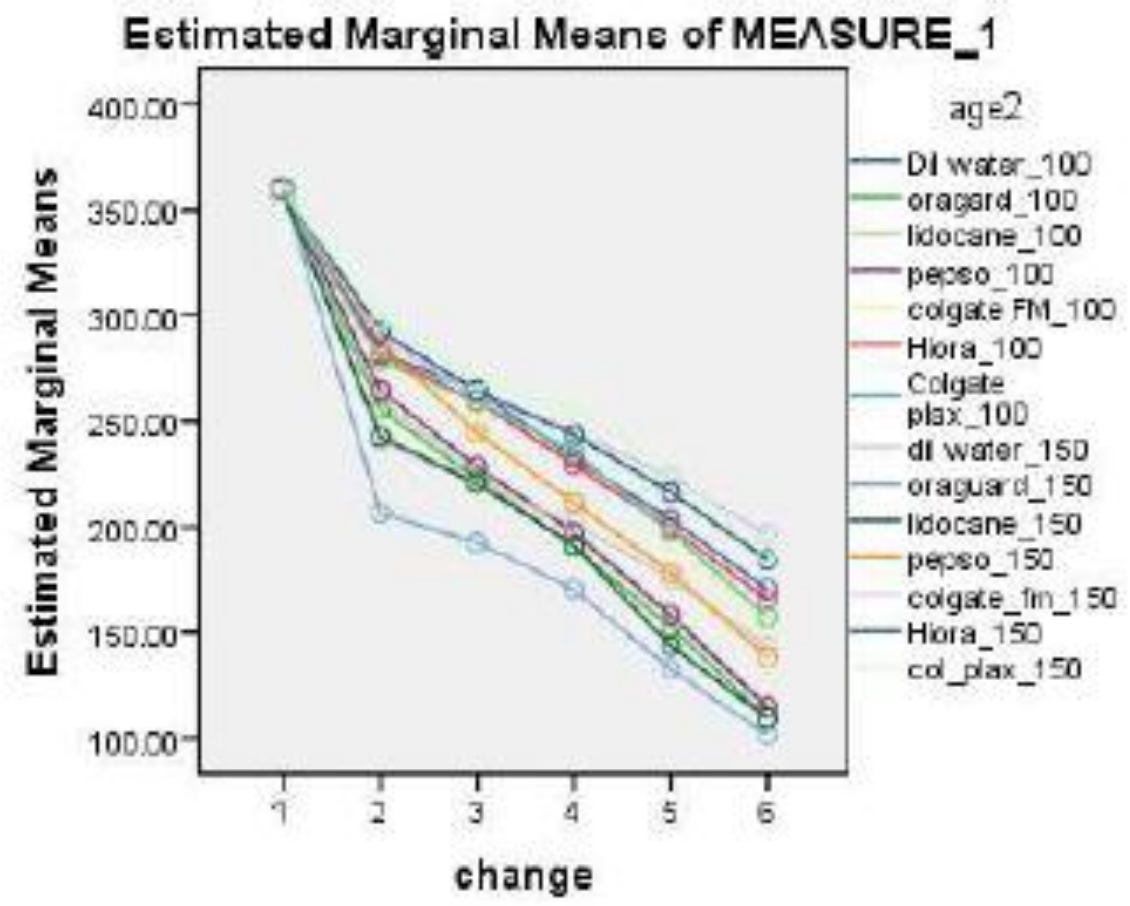

Table: Inter group stafistical analysis between the Test Groups (thers Group Comparison) of both Sample A and Sample B.

\begin{tabular}{|c|c|c|c|c|c|}
\hline Sowase & $\begin{array}{c}\text { Trpe II Sate of } \\
\text { Squars }\end{array}$ & af & Mesel Siquere & F & Sis \\
\hline Chesere & $115 \operatorname{sas} \operatorname{ses} 3$ & s & 200053.165 & 48173022.124 & .000 \\
\hline 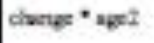 & $3900 \mathrm{k}$. $44 \mathrm{~s}$ & 65 & staioss & 10sises 014 & 000 \\
\hline Pront(duarye) & 690 & 140 & .005 & & \\
\hline
\end{tabular}


Graph 2:- Force decay patterns (In percentage) observed when comparisons were done between the Test Groups (Inter Group Comparison) of both Sample A and Sample B

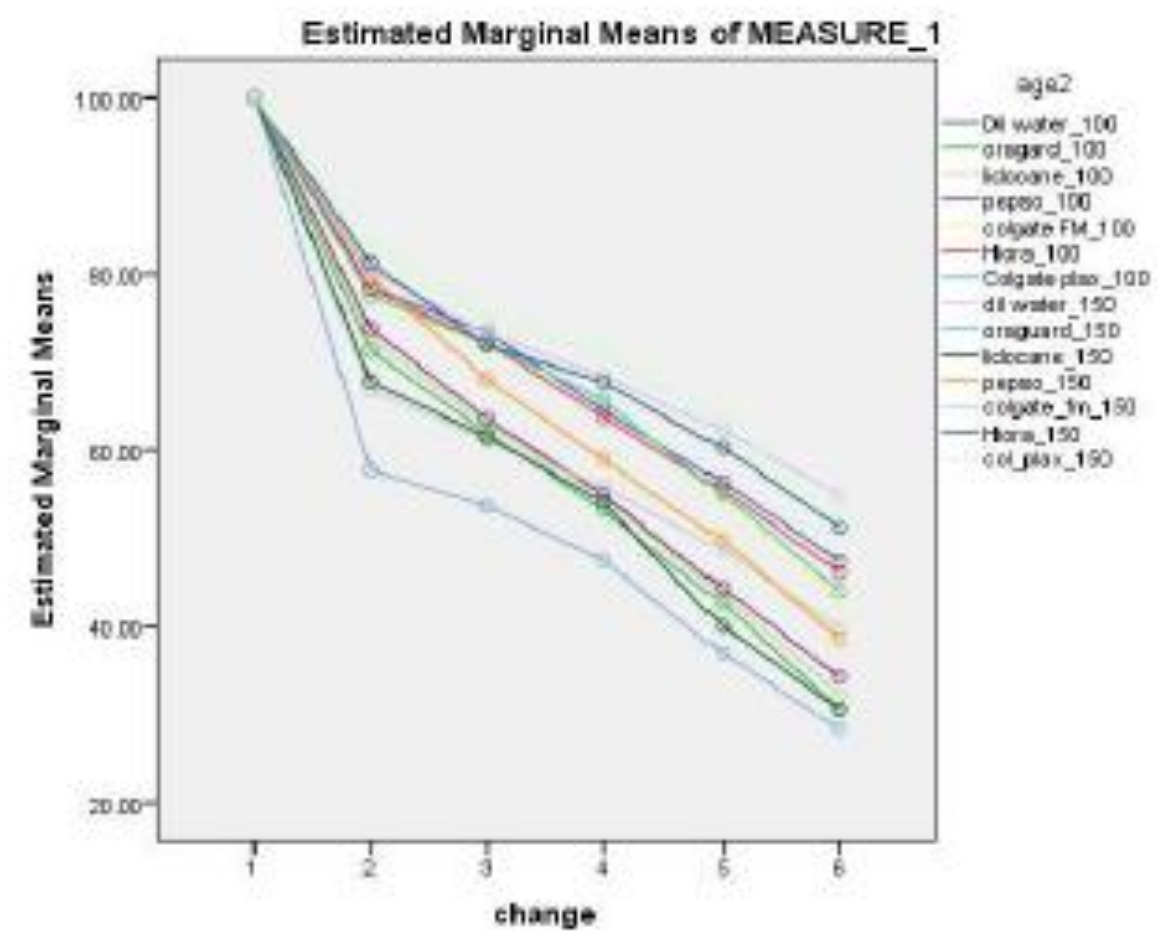

Teat of Witin-Sabject Entein

Mhase: MFAsSURE_1

\begin{tabular}{|c|c|c|c|c|c|}
\hline Sceurx & $\begin{array}{c}\text { Type III Sun of } \\
\text { Squen }\end{array}$ & dr & Mecen Square & f & 85 \\
\hline Chene & $8500 \times 329$ & 5 & 1 1\%N2.66t & 51697.74 & 600 \\
\hline change + age 2 & 2512660 & 65 & 4n:185 & 116711 & $\operatorname{sic}$ \\
\hline Pinorictuage) & 48256 & 140 & 344 & & \\
\hline
\end{tabular}


Graph 3:- Force decay patterns (In percentage) observed when comparisons were done between Sample A and Sample B (Intra Group Comparison)

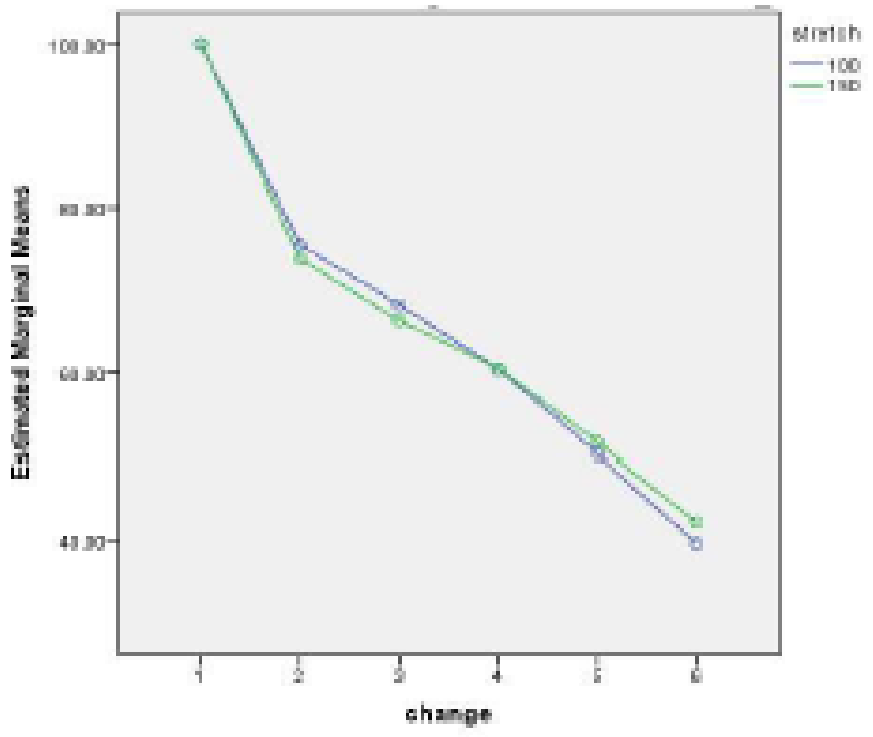

\section{Discussion:-}

Elastomeric orthodontic chain, in general, are polyurethanes; thermosetting polymer products of a step-reaction polymerization process, possessing a - OCONH- unit1. Polyurethane elastomers are produced by the rearrangement polymerization of di-isocyanates and polyols 2 . The elastomeric polymer is easily stretched and substantially regains its shape on relaxation in acidic or neutral $\mathrm{pH}$. The issue of force decay pattern has received increased interest since polyurethanes are not inert materials and are highly affected by heat, moisture and prolonged contact with enzymes. Mouthwashes are commonly used by orthodontic patients and elastomeric chains were pre-stretched to bring down the force levels to within physiologic limits; thus, it is vital to investigate how mouthwashes and pre-stretch incorporated in elastomeric chains affect force decay.

This study tested force decay of pre-stretched elastomeric chains in mouth washes containing alcohol and without alcohol. The fact that there is increased prevalence of gingivitis and development of white spot lesions during fixed orthodontic treatment, incorporating a chemotherapeutic agent, such as an antibacterial mouth rinse, into the oral hygiene procedures to improve plaque retention is often recommended. Many of these mouth rinses contain ethanol as solvent in various concentrations. The results of this study demonstrate that pre-stretching elastomeric chain to $100 \%$ the initial length has more force decay than the one stretched $150 \%$. Alcohol causes a significant increase in the amount of force decay seen in elastomeric chains exposed to commercial mouth rinses containing alcohol compared to those exposed only to water. The results also show force decay over time which is high initially during the first one hour and then progresses gradually over the rest of the period of 4 weeks. For all Groups the greatest force decay occurred during the first One hour of Immersion in the media15. The result of this study showed a force decay of 21.4\% for Deionised water, $28.6 \%$ for Alcoholic 21.6\%v/v Oragard Mouth rinse, $26.8 \%$ for Alcoholic $10 \% \mathrm{v} / \mathrm{v}$ Lidocam mouth rinse, $26.2 \%$ for Alcoholic 6\%v/v Pepsodent Mouth rinse, $22.7 \%$ for Non Alcoholic Freshmint Mouth rinse, 21.7\% for Non Alcoholic Hiora-k Mouth rinse, 22.4\% for Non Alcoholic Sensitive mouth rinse in the first 1 hour. The Control media Group showed greatest amount of decay occurring in the first 1 hour. This is in agreement with the previous studies on force decay of elastomeric chains. The decay progressed gradually from 0 days till 28 days until $47.35 \%$ and 39.08\% of Initial force was remaining at the end of the 4th week in Sample A and Sample B respectively. The force decay pattern of e-chains in Deionised water is consistent with the force decay pattern of elastomeric chains as evidenced by the previous studies. Comparing the force decay patterns of DI water and Alcoholic groups, the residual force at the end of $4^{\text {th }}$ week of elastomeric chains immersed in deionised water is comparatively higher and statistically significant $(\mathrm{p}=0.001)$ than the residual force at the end of 4th week of elastomeric chains immersed in Alcoholic Mouth rinses . The Alcoholic groups (Media B1, B2, and B3) too showed greatest force decay occurring in the first One Hour of Immersion in the media. The force decay progressed gradually from 0 days until the amount of remaining force at the end of $4^{\text {th }}$ week was between $30.6 \%$ $34.18 \%$ and $28.27-38.56 \%$ of the Initial Force in each groups Sample A and Sample B respectively (Table and 
graph). This amounts to $109.54 \mathrm{gms}$ and $101.57 \mathrm{gms}$ of force respectively at the end of 4 th week. The force decay was significantly higher $(\mathrm{P}=0.001)$ for the alcoholic group at each time points when compared to both the control media group and also to the Non Alcoholic Group. This increased force decay can be attributed to ethanol present in the alcoholic mouth rinses as a dissolvent and a carrier for active ingredients. When analyzed microscopically, immersion of polyurethane elastomeric modules in a $75 \%$ ethanol/water mixture caused structural and molecular modifications leading to decay of specimen. Between the Alcoholic Groups studied, the Force decay pattern was similar with resultant force values that were similar and not statistically significant $(\mathrm{p}=0.746, \mathrm{p}=0.138, \mathrm{p}=0.834)$, indicating that concentration of alcohol doesn't seem to affect the magnitude of force decay in elastomeric chains. This is in agreement to the previous study on the effect of alcoholic concentration on the force decay of elastomeric chains. The Non Alcoholic Groups (Media C1, C2, and C3) showed similar patterns of force decay exhibiting greatest force decay in the first One hour of Immersion in the Media.

The force decay progressed gradually from 0 days until the amount of remaining force at the end of 4 th week in ach test samples of Sample A and Sample B was between 43.02-43.96\% and52.35-54.83\% respectively of the Initial force which amounts to $154.41-157.5 \mathrm{Gms}$ and $186.56-196.5 \mathrm{Gms}$ respectively of force at the end of 4th week. This residual force is significantly higher than the residual force of alcoholic group (30.6\%-34.18\% and 28.27$38.56 \%)(\mathrm{p}=0.001)$ suggesting that Force decay occurred to a much lesser amount in e-chain stretched $150 \%$ and Non Alcoholic mouth rinse group as compared to e-chains stretched $100 \%$ and Alcoholic Mouth rinses Group. The pattern of force decay in Non Alcoholic mouth rinse media was slightly lesser to the force decay patterns of Control Deionised water with the Deionised water group showing a residual force value of $47.35 \%$ and $39.08 \%$ and Non Alcoholic group showing 43.02- 43.96\% and 52.35-54.83\% which was statistically significant ( $p=0.001)$ but not clinically significant. Among the Non Alcoholic groups studied, the force decay pattern was similar within the groups however Hiora-k Mouth rinse exhibited least effect on force decay on the elastomeric chains as compared to other Non Alcoholic mouth rinses Studied( $\mathrm{P}=0.001)$. Thus it can be safely assumed that the effect of pre-stretching the e-chain to $150 \%$ has less force decay and Non Alcoholic mouth rinses produce an amount of force decay slightly higher or similar to Deionised water. Therefore pre-stretching the e chain to $150 \%$ for 10 seconds prior to usage would have better and longer effect than its counterpart stretched double or $100 \%$ initial length, and the prescription of Non Alcoholic mouth rinses by Orthodontists would be beneficial to maintain a higher amount of force in elastomeric chains for effective tooth movement during the phase of retraction when patients are frequently seen at a 4 week gap for reactivation. Until research can demonstrate otherwise, the current practice of changing elastomeric chains every 21-28 days during tooth movements seems acceptable from the perspective of performance of dental materials.

\section{Conclusion:-}

The force decay patterns of Control Deionsed water group and Non Alcoholic Mouth rinses group in $150 \%$ prestretched e-chain was comparable and prescription of Non Alcoholic mouthwashes seems optimum for hygiene maintenance as compared to Alcoholic mouth rinses in order to have the least effect on force decay of elastomeric chains.

\section{Bibliography:-}

1. Parrie WJ, Spence JA. Elastics - their properties and clinical applications inorthodontic fixed appliance therapy. Br J Orthod 1973;1:167-71

2. Billmeyer FW. Textbook of polymer science.3rd ed. New York :JohnWiley,1984

3. Haper CA. Handbook of plastics and elastomers. New York: McGraw-Hill,1975.

4. Andreasen GF, Bishara SE. Comparison of alastik chains and elastics involvedwith intra-arch molar to molar forces. Angle Orthod 1970; 40:151-8.

5. Hershey G, Reynolds W. The plastic module as an orthodontic tooth movingmechanism. Am J Orthod 1975; 67: 554-662

6. Wong A. Orthodontic elastic materials. Angle Orthod 1976; 46:196-205 